\section{A practical approach to the chest $x$-ray of a child with known or suspected congenital heart disease}

- The state of the pulmonary vasculature

- The position and shape of the aortic arch

- The cardiac and visceral situs

- Skeletal abnormalities

- Evidence of cardiac failure

- Evidence of infection

\section{The size and shape of the heart}

It is generally accepted that the normal cardiothoracic ratio (CTR) between nought and two years of age is $60 \%$ and that over the age of four years it should be $50 \%$. It stands to reason that the CTR between the ages of two and four is between $50 \%$ and $60 \%$. An enlarged heart may be the only sign of CHD early on, highlighting the importance of measuring the CTR accurately (see Figure 1).

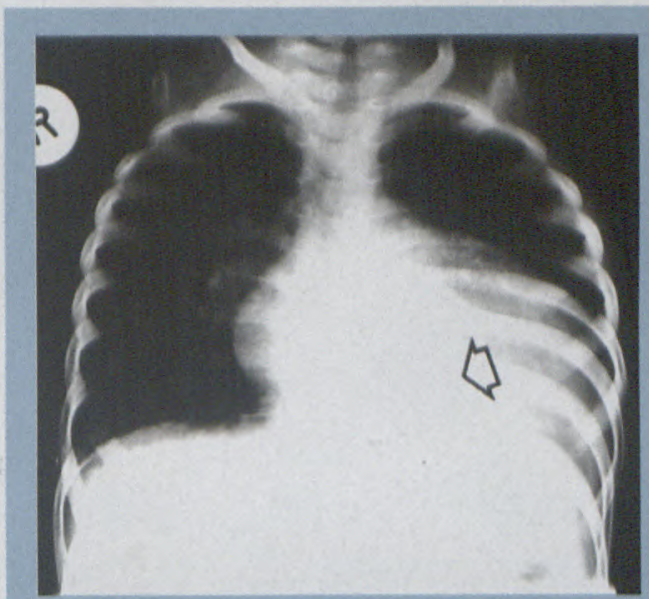

Figure 1: Gross cardiomegaly is noted in this twoyear-old patient with a cardio-thoracic ratio of $>60 \%$. Note the associated LLL collapse caused by compression of the left main bronchus (open arrow).

Many imaginative characteristic descriptions of specific anomalies have been made, e.g. the boot-shaped heart of Tetralogy of Fallot (see Figure 2), the snowman of TAPVD (totally anomalous pulmonary venous drainage) or the egg-shaped heart of uncorrected transposition of the great 


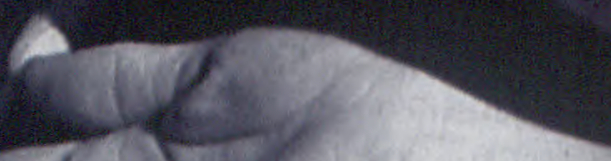




\section{Gadopentetate dimeglumine}
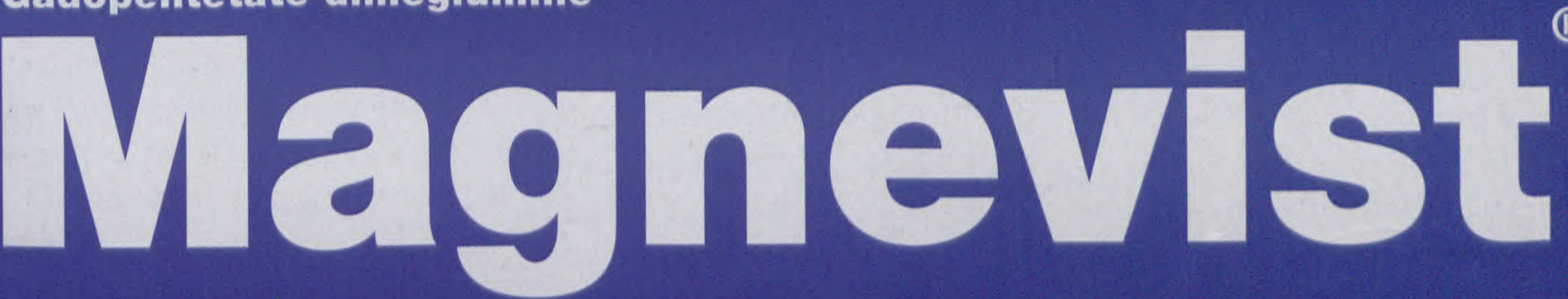


\section{A practical approach to the chest $x$-ray of a child with known or suspected congenital heart disease}

from page 31

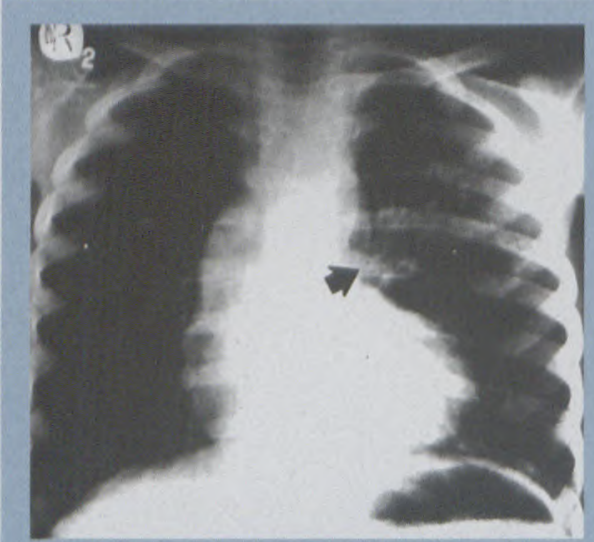

Figure 2: This patient with Tetralogy of Fallot demonstrates right ventricular cardiomegaly and "hollow pulmonary bay" (closed arrow), giving the typical "boot-shaped" heart (coeur-en-sabot). Note the oligaemic right lung as compared with the normally

vascularised left lung, which had been shunted.

vessels. These characteristic shapes occur only in the minority of examples of each anomaly. The snowman appearance of TAPVD can easily be caused by the thymic shadow of the young child. Also, with early surgery, these characteristic shapes are now rarely seen.

\section{The state of the pulmonary vasculature}

The assessment of the pulmonary vasculature is extremely important, but unfortunately remains quite subjective and requires good quality radiographs for the observer to be confident. The radiologist needs to decide if the lungs are oligaemic (decreased pulmonary blood flow) or plethoric (increased pulmonary blood flow). One easy method of noting plethora is to obliterate the medial two-thirds of each lung; if vessels are easily noted in the outer third, there is likely to be increased pulmonary blood flow (see Figure 3a). An additional finding commonly noted in patients with increased pulmonary blood flow is diffuse hyperinflation of the lungs with sloping of and flattening of the

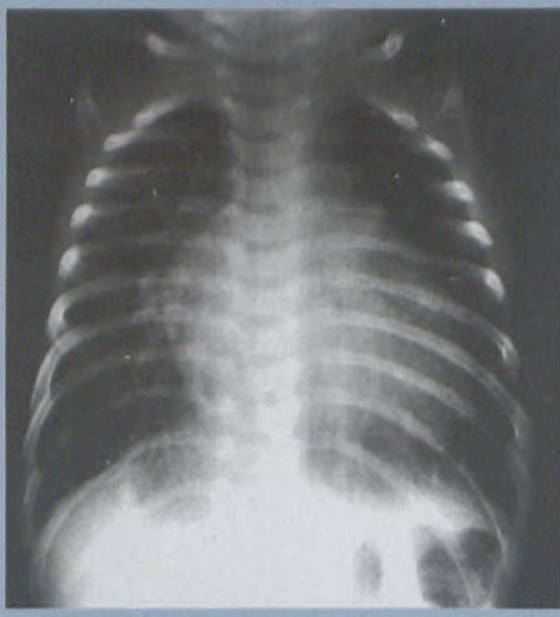

Figure 3a: The patient with an AVSD shows prominent pulmonary vessels extending to the periphery in keeping with plethora due to the leftto-right shunt

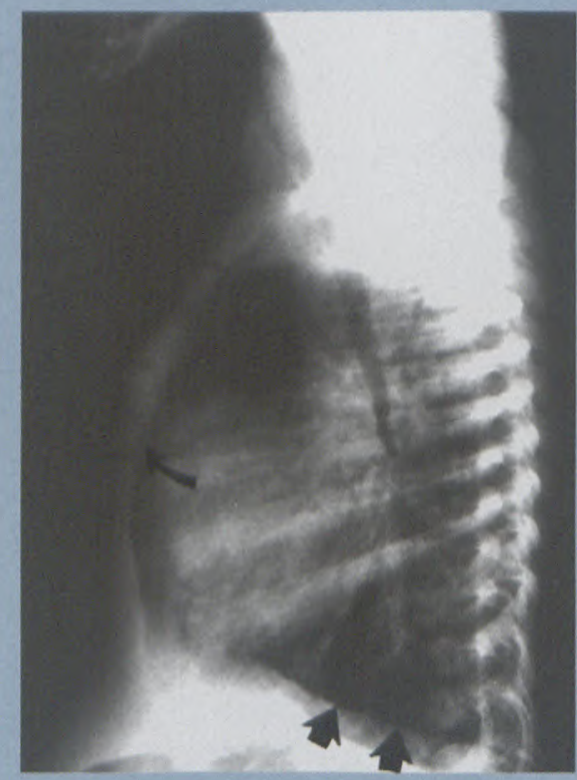

Figure 3b: The lateral $X$-ray of the same patient demonstrates the radiological features of air-trapping associated with a left-to-right shunt, i.e. flattening of the hemidiaphragms (closed arrows) and bowing of the anterior chest wall (curved arrow)

hemidiaphragms, anterior bowing of the chest wall and widening of the intercostal spaces (see Figure 3b). The state of the pulmonary vasculature in conjunction with the colour of the patient, i.e. cyanotic or acyanotic (which is usually noted on the request form), forms the basis of our classification in the table.

\section{Classification of CHD and the more common anomalies}

\section{ACYANOTIC}

- With L to R shunt: atrial septal defect, ventricular septal defect, patent ductus arteriosus

- Without L to R shunt: pulmonary stenosis, aortic stenosis, coarctation of the aorta

\section{CYANOTIC}

- With increased pulmonary flow: transposition of the great vessels, total anomalous pulmonary venous drainage, truncus arteriosus

\section{- With decreased pulmonary flow:} Tetralogy of Fallot, tricuspid atresia, double outlet right ventricle and pulmonary stenosis, Ebsteins anomaly

\section{Position of the aortic arch}

The importance of assessing the position of the aortic arch lies in its association with specific cardiac anomalies as well as the planning of shunt procedures. A right-sided aortic arch is found in 25\% of Tetralogy of Fallot, 25\% of DORV (double-outlet right ventricle), $35 \%$ of truncus arteriosis and $12,5 \%$ of single-ventricle anomalies.

The position of the arch is often not easy to assess in the young child. One useful hint is to note the position of the trachea, which is normally slightly deviated to the right in the child. If the trachea is central or displaced to the left, one should suspect a right-sided aortic arch (see Figure 4).

An important observation is that the shape of the aortic arch is often abnormal in coarctation. 


\section{from page 34}

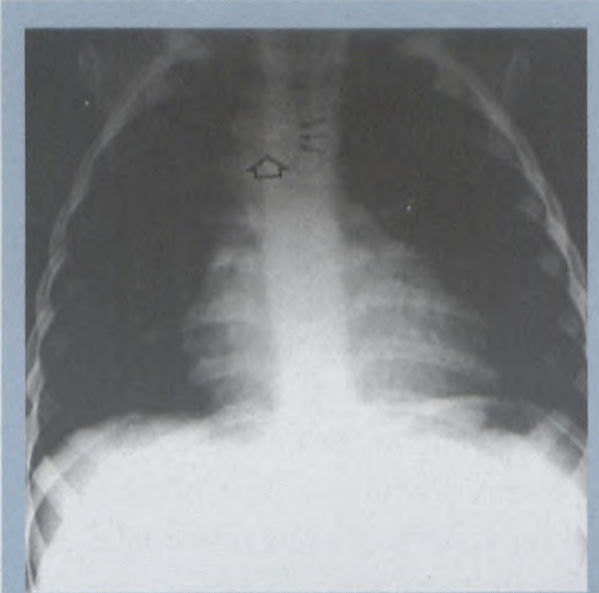

Figure 4: This two-year-old patient has a rightsided aortic arch (open arrow). Note the central position of the trachea and the impression on its lower border by the aorta (small arrows).

\section{Cardiac and visceral situs}

The incidence of CHD is high in patients with dextrocardia and normally sited abdominal viscera. The incidence of $\mathrm{CHD}$ in patients with true situs inversus (heart and abdominal viscera on reversed side) is much lower and, in fact, is almost the same as that for the normal population (see Figure 5).

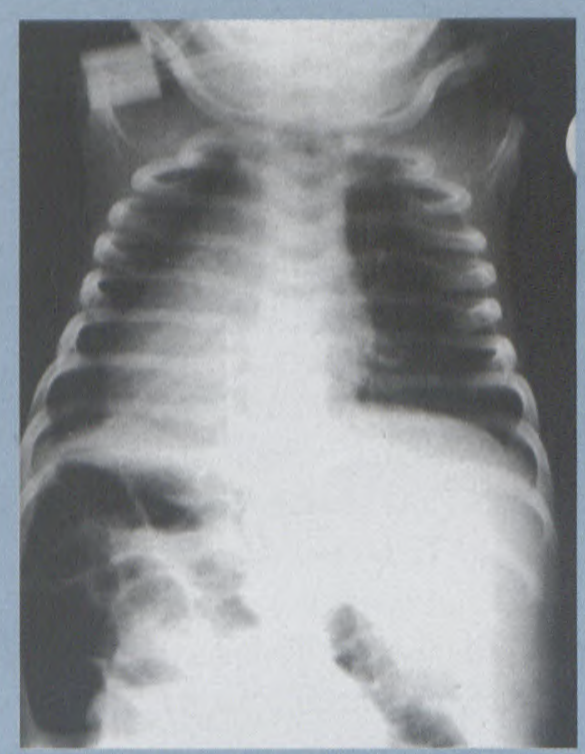

Figure 5: In patients like this one-month-old infant with true situs inversus, there is a low incidence of CHD. This is in contrast to patients with dextrocardia or levocardia with abdominal situs abnormalities, who have a high incidence of CHD.

\section{Skeletal abnormalities}

A most important observation to be made is to assess for evidence of previous surgery. A sternotomy usually means that the cardiac defect has been corrected. A thoracotomy is usually done for one of the following procedures: pulmonary artery banding, Blallock/modified Blallock shunt (see Figure 6), closure of a PDA or repair of a coarctation of the aorta.

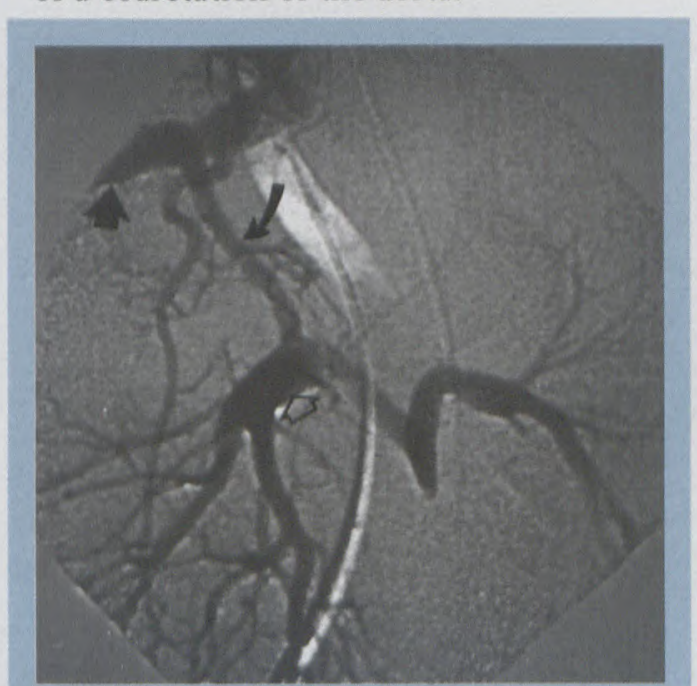

Figure 6: This digital subtration angiogram demonstrates a modified Blallock shunt (curved arrow) connecting the right subclavian artery (closed arrow) with the right pulmonary artery (open arrow). Note the improved vascularisation of the right lung.

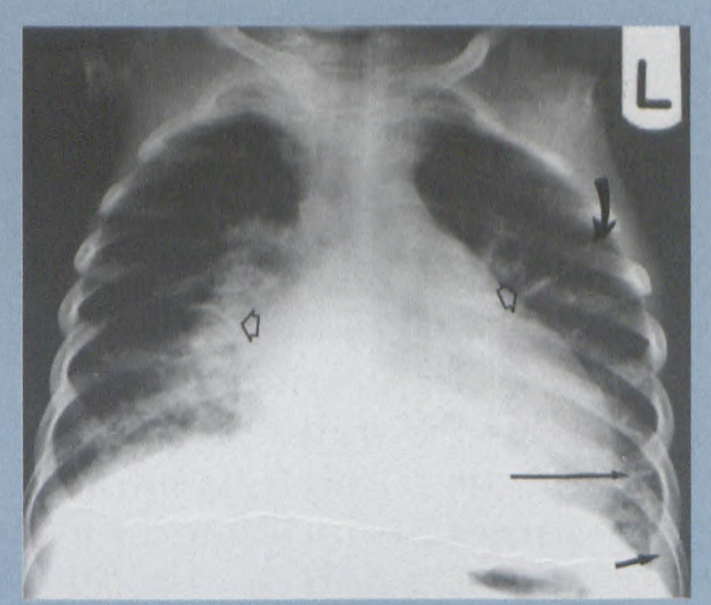

Figure 7: This chest $X$-ray demonstrates the features of cardiac failure in a three-year-old patient with CHD. Note the cardiomegaly, pleural effusion (short arrow), peri-hilar haze (open arrow) and both Kerley $A$ (curved arrow) and Kerley B (long arrow) lines.
Notching of the ribs can be noted in the older child (usually after the age of 10 years) with coarctation of the aorta. This can also be a feature of the child with a long-standing Blallock shunt.

One should always remember that the cardiac lesion may be part of a syndrome or a complex of anomalies. An example of a complex of anomalies is the VACTREL (vertebral, anorectal, cardiac, tracheal, renal, oesophageal, limb) association. Common syndromes include Down's syndrome (trisomy 21), which is associated with endocardial cushion defects, VSDs and Tetralogy of Fallot, and Turner's syndrome, which is associated with aortic coarctation.

\section{Evidence of cardiac failure}

The features of cardiac failure on the chest X-ray should be actively sought. These features include upper-lobe blood diversion, peri-hilar haze, pleural effusions and septal lines (see Figure 7). In children, Kerley A lines are often more prominent than Kerley B lines, unlike the situation in adults.

The importance of cardiac failure lies in the fact that it has some prognostic value. For example, a lone ASD or a VSD with a shunt less than 2:1 (pulmonary to systemic flow) is unlikely to cause cardiac failure early on, whereas a larger left to right shunt having a worse prognosis will cause cardiac failure sooner. 